TRANSACTIONS OF THE

AMERICAN MATHEMATICAL SOCIETY

Volume 365, Number 7, July 2013, Pages 3575-3591

S 0002-9947(2013)05682-6

Article electronically published on January 17, 2013

\title{
RANDOMNESS FOR NON-COMPUTABLE MEASURES
}

\author{
ADAM R. DAY AND JOSEPH S. MILLER
}

\begin{abstract}
Different approaches have been taken to defining randomness for non-computable probability measures. We will explain the approach of Reimann and Slaman, along with the uniform test approach first introduced by Levin and also used by Gács, Hoyrup and Rojas. We will show that these approaches are fundamentally equivalent.

Having clarified what it means to be random for a non-computable probability measure, we turn our attention to Levin's neutral measures, for which all sequences are random. We show that every PA degree computes a neutral measure. We also show that a neutral measure has no least Turing degree representation and explain why the framework of the continuous degrees (a substructure of the enumeration degrees studied by Miller) can be used to determine the computational complexity of neutral measures. This allows us to show that the Turing ideals below neutral measures are exactly the Scott ideals. Since $X \in 2^{\omega}$ is an atom of a neutral measure $\mu$ if and only if it is computable from (every representation of) $\mu$, we have a complete understanding of the possible sets of atoms of a neutral measure. One simple consequence is that every neutral measure has a Martin-Löf random atom.
\end{abstract}

\section{DEFining RANDOMNESS}

Let $X$ be an element of Cantor space and $\mu$ a Borel probability measure on Cantor space. What should it mean for $X$ to be random with respect to $\mu$ ? In the case that $\mu$ is the Lebesgue measure, then the theory of $\mu$-randomness is well developed (for recent treatises on the subject the reader is referred to Downey and Hirschfeldt, and Nies 2, 13]).

In fact if $\mu$ is a computable measure, then early work of Levin showed that $\mu$-randomness can be seen as essentially a variant on randomness for Lebesgue measure [10]. This leaves the question of how to define randomness if $\mu$ is noncomputable. We will show that the two approaches that have previously been used to define $\mu$-randomness, for non-computable $\mu$, are equivalent. Later, in Theorem 4.12, we will provide another characterization of $\mu$-randomness using the enumeration degrees.

For Lebesgue measure, the preeminent definition of randomness was originally provided by Martin-Löf and is now known as Martin-Löf randomness 11. We would like to find a natural generalization of Martin-Löf randomness to non-computable probability measures. One approach is to generalize Martin-Löf tests. This approach immediately runs into the difficult question of what sort of oracle access a test should have. It is reasonable to expect that a test for a measure $\mu$ should be able

Received by the editors November 21, 2010 and, in revised form, August 10, 2011.

2010 Mathematics Subject Classification. Primary 03D32; Secondary 68Q30, 03D30.

The second author was supported by the National Science Foundation under grants DMS0945187 and DMS-0946325, the latter being part of a Focused Research Group in Algorithmic Randomness. 
to compute the $\mu$ measure of any basic clopen set. However, there are continuum many probability measures on Cantor space, so in order to make these measures accessible to the techniques of computability theory, we will make use of some basic concepts of computable analysis. We will define all the concepts we need. For further background on computable analysis, the reader is referred to Weihrauch [19], who gives a modern development of the subject. Classical computability theory studies Cantor space $\left(2^{\omega}\right)$ and Baire space $\left(\omega^{\omega}\right)$. The main idea behind computable analysis is to transfer the notions of computability theory to other structures via representations of those structures. If $\mathcal{S}$ is a set, a representation of $\mathcal{S}$ is just a surjective function (possibly partial) $\rho: 2^{\omega} \rightarrow \mathcal{S}$. The representation induces a computability-theoretic structure on $\mathcal{S}$. We will also use the word "representation" in another, less standard, sense. If $R \in 2^{\omega}$ and $\rho(R)=x$, we call $R$ a representation of $x$.

We will take $\mathcal{P}\left(2^{\omega}\right)$ to be the set of all probability measures on Cantor space. We will let $\rho: 2^{\omega} \rightarrow \mathcal{P}\left(2^{\omega}\right)$ be a representation of $\mathcal{P}\left(2^{\omega}\right)$. In Section 2 we will give a detailed definition of such a $\rho$, but for now it is enough to specify that if $\rho(R)=\mu$, then we can uniformly in $R$ compute the $\mu$-measure of any basic clopen set in Cantor space.

As we access measures via representations, one approach is to define randomness in terms of representations. The following definitions, while not identical, are equivalent to that of Reimann [14] and Reimann and Slaman [15].

Definition 1.1. Let $\mu \in \mathcal{P}\left(2^{\omega}\right)$ and let $R \in 2^{\omega}$ be a representation of $\mu$.

(i) An $R$-test is a uniform (in $R$ ) sequence $\left\{V_{i}\right\}_{i \in \omega}$ of $\Sigma_{1}^{0}(R)$ sets such that $\mu\left(V_{i}\right) \leq 2^{-i}$.

(ii) $X \in 2^{\omega}$ passes an $R$-test if $X \notin \bigcap_{i} V_{i}$.

(iii) $X \in 2^{\omega}$ is $R$-random if it passes all $R$-tests.

A universal $R$-test exists for the same reason that a universal Martin-Löf test exists. Given $R$, we would like to enumerate all $R$-tests by enumerating all (uniform in $R$ ) sequences of $R$-c.e. sets, halting any enumeration if it would exceed the measure bound. There is a small technical obstruction, namely that we cannot exactly compute the $\mu$-measure of a basic clopen set from $R$. However, we can compute a sequence approximating it from above. Hence, we can pause an enumeration until a stage when our approximation from above guarantees that we can add the next element without exceeding the measure bound. Note that this could cause a problem if some test $\left\{V_{n}^{R}\right\}_{n \in \omega}$ had $V_{i}^{R}=2^{-i}$ for some $i$. The enumeration of this test could be paused forever. However, in this case, the test $\left\{V_{n+1}^{R}\right\}_{n \in \omega}$ defines the same null set and avoids this problem. This shows that we can (essentially) enumerate all $R$-tests, uniformly in $R$, so we can build a universal $R$-test. Even better, because the construction is uniform, there is a uniform sequence of c.e. sets $U_{n}$ such that if $U_{n}^{R}=\left\{[\tau]:\langle\tau, \sigma\rangle \in U_{n}\right.$ and $\left.\sigma \prec R\right\}$, then $\left\{U_{n}^{R}\right\}_{n \in \omega}$ is a universal $R$-test. Call $\left\{U_{n}\right\}_{n \in \omega}$ a universal oracle Martin-Löf test. Our notation is standard: $\sigma$ and $\tau$ are used for finite binary strings (elements of $2^{<\omega}$ ); $\sigma X$ is $\sigma$ concatenated with $X ;[\sigma]$ is the basic clopen set $\left\{\sigma X: X \in 2^{\omega}\right\} ; \sigma \prec X$ holds if $\sigma$ is an initial segment of $X$.

As noted by Reimann, the problem with Definition 1.1 is that it is dependent on the representation. Given any measure, it is possible to encode any sequence into some representation of that measure. Hence for all $\mu \in \mathcal{P}\left(2^{\omega}\right)$ and all $X \in 2^{\omega}$, 
there is a representation $R$ of $\mu$ such that $X$ is not $R$-random. A natural way to overcome this problem is with the following definition.

Definition 1.2. A sequence $X \in 2^{\omega}$ is $\mu$-random if there exists a representation $R$ of $\mu$ such that $X$ is $R$-random.

Our goal is to show that, at least in Cantor space, this definition gives the same class of randoms for a measure as does the concept of a uniform test. Uniform tests are an alternative approach to randomness for non-computable measures. They were introduced by Levin and developed by Gács, and Hoyrup and Rojas [3, 4, 9. While uniform tests can be applied to general probability spaces, in this paper, we will only be concerned with Cantor space.

Definition 1.3. Consider a function $t: \mathcal{P}\left(2^{\omega}\right) \times 2^{\omega} \rightarrow \mathbb{R}^{\geq 0} \cup\{\infty\}$.

(i) The under-graph of $t$ is $\{(\mu, X, r): t(\mu, X)>r\}$. We say that the undergraph of $t$ is c.e. open if it is equal to $\bigcup_{\langle i, \sigma, q\rangle \in W} B_{i} \times[\sigma] \times[0, q)$ for some c.e. set $W \subseteq \omega \times 2^{<\omega} \times \mathbb{Q}$.

(ii) We call $t$ a uniform test if its under-graph is c.e. open and for every $\mu \in$ $\mathcal{P}\left(2^{\omega}\right)$ we have $\int t(\mu, X) d \mu \leq 1$.

(iii) $X \in 2^{\omega}$ passes a test $t$ for a measure $\mu$ if $t(\mu, X)$ is bounded.

(iv) $X \in 2^{\omega}$ is $\mu$-random for uniform tests if it passes all tests for measure $\mu$.

By a straightforward theorem of Gács, later refined by Hoyrup and Rojas, it is sufficient to consider a single universal uniform test.

Definition 1.4. A uniform test $t$ is universal if for all uniform tests $t^{\prime}$ there is a constant $c>0$ such that for all $\mu \in \mathcal{P}\left(2^{\omega}\right)$ and $X \in 2^{\omega}$, we have $t(\mu, X) \geq c t^{\prime}(\mu, X)$.

Theorem 1.5 (Gács; Hoyrup and Rojas [3, 4]). There exists a universal uniform test.

The following theorem will establish the equivalence of these two approaches.

Theorem 1.6. For any measure $\mu$ and $X \in 2^{\omega}$ we have that $X$ is $\mu$-random if and only if $X$ is $\mu$-random for uniform tests.

Before proving this theorem, we need to take a more detailed look at $\mathcal{P}\left(2^{\omega}\right)$ and at representations of probability measures.

\section{Non-COMputable Probability measures}

In this paper, we will restrict our investigation to Borel probability measures on Cantor space. Let $\mu$ be such a measure. We can identify $\mu$ with the values it takes on the basic clopen sets $[\sigma]$, where $\sigma \in 2^{<\omega}$. Hence we will often think of $\mu$ as a function $\mu: 2^{<\omega} \rightarrow \mathbb{R}^{\geq 0}$ and write $\mu(\sigma)$ instead of $\mu([\sigma])$. Take $\left\langle_{-}\right\rangle: 2^{<\omega} \rightarrow \omega$ to be the standard bijection between $2^{<\omega}$ and $\omega$ (i.e., the mapping that takes $\lambda, 0,1,00$, and 01 to $0,1,2,3$, and 4 respectively). Any measure $\mu$ such that $\int d \mu \leq 1$ can be thought of as an element $\alpha \in[0,1]^{\omega}$, where $\alpha(\langle\sigma\rangle)=\mu(\sigma)$. We define the following two subspaces of $[0,1]^{\omega}$ :

(i) $\mathcal{M}\left(2^{\omega}\right)=\left\{\alpha \in[0,1]^{\omega}:\left(\forall \sigma \in 2^{<\omega}\right) \alpha(\langle\sigma\rangle)=\alpha(\langle\sigma 0\rangle)+\alpha(\langle\sigma 1\rangle)\right\}$,

(ii) $\mathcal{P}\left(2^{\omega}\right)=\left\{\alpha \in \mathcal{M}\left(2^{\omega}\right): \alpha(\langle\lambda\rangle)=1\right\}$.

Our primary space of concern is $\mathcal{P}\left(2^{\omega}\right)$, the space of all probability measures on Cantor space. The space $\mathcal{M}\left(2^{\omega}\right)$, all measures $\mu$ such that $\int d \mu \leq 1$, will be of 
interest when we investigate neutral measures. We can regard $\mathcal{P}\left(2^{\omega}\right)$ and $\mathcal{M}\left(2^{\omega}\right)$ as compact subspaces of the topological vector space $\mathbb{R}^{\omega}$ with the topology provided by the metric

$$
d(\alpha, \beta)=\sum_{\sigma \in 2^{<\omega}} 2^{-\langle\sigma\rangle}|\alpha(\langle\sigma\rangle)-\beta(\langle\sigma\rangle)|
$$

Additionally, $\mathcal{M}\left(2^{\omega}\right)$ and $\mathcal{P}\left(2^{\omega}\right)$ are both convex subspaces of $\mathbb{R}^{\omega}$ (where $C$ is convex if for all $\mu, \nu \in C$ and $x \in[0,1]$, we have $x \mu+(1-x) \nu \in C)$.

There is an alternative approach to topologizing the space $\mathcal{P}\left(2^{\omega}\right)$. We can topologize this space so that a sequence of measures $\left\{\mu_{n}\right\}_{n \in \omega}$ has limit $\mu$ if and only if $\mu_{n}(B) \rightarrow \mu(B)$ for all Borel sets $B$ whose boundary has $\mu$ measure 0 . This topology is known as the weak topology.

We can view $2^{\omega}$ as a metric space by using the metric

$$
d_{2^{\omega}}(A, B)= \begin{cases}0 & \text { if } A=B, \\ 2^{-i} & \text { where } i=\min \{A \triangle B\} \text { otherwise. }\end{cases}
$$

Cantor space is a compact and separable metric space under $d_{2^{\omega}}$. This fact implies that the weak topology on $\mathcal{P}\left(2^{\omega}\right)$ is compact and further that it is metrizable using the Prohorov metric [1]. Given $\mu, \nu \in \mathcal{P}\left(2^{\omega}\right)$, the Prohorov metric $p(\mu, \nu)$ is defined to be the infimum of those positive $\epsilon$ for which the following two inequalities hold for all Borel subsets $A$ of $2^{\omega}$ :

$$
\mu(A) \leq \nu\left(A^{\epsilon}\right)+\epsilon, \quad \nu(A) \leq \mu\left(A^{\epsilon}\right)+\epsilon,
$$

where $A^{\epsilon}=\left\{X \in 2^{\omega}:(\exists Y \in A) d(X, Y)<\epsilon\right\}$.

Note that, under the metric $d_{2^{\omega}}$, if $A \subseteq 2^{\omega}$ and $\epsilon=2^{-n}$, then $A^{\epsilon}=\bigcup\{[\sigma]:|\sigma|=$ $n \wedge[\sigma] \cap A \neq \emptyset\}$. Using this observation, the following lemma is easy to show.

Lemma 2.1. The Prohorov metric and the metric defined in (2.1) induce the same topologies on $\mathcal{P}\left(2^{\omega}\right)$.

Proof. Let $p$ be the Prohorov metric and $d$ the metric as defined in (2.1). Pick any $\mu \in \mathcal{P}\left(2^{\omega}\right)$ and positive real number $\delta$. Now consider the open ball using the Prohorov metric $B_{p}(\mu, \delta)$. Choose $n \in \omega$ such that $2^{-n}<\delta$. Choose $\epsilon$ so that for all $\nu \in B_{d}(\mu, \epsilon)$ (the open ball using the metric $d$ ) we have that for all $\sigma$ of length $n,|\mu(\sigma)-\nu(\sigma)|<2^{-2 n}$. Then if $A \subseteq 2^{\omega}$ is Borel we have that

$$
\mu A \leq \mu A^{2^{-n}}=\sum_{\substack{|\sigma|=n \\[\sigma] \cap A \neq \emptyset}} \mu(\sigma)<\sum_{\substack{|\sigma|=n \\[\sigma] \cap A \neq \emptyset}}\left(\nu(\sigma)+2^{-2 n}\right)=\nu A^{2^{-n}}+2^{-n} .
$$

Similarly $\nu A<\mu A^{2^{-n}}+2^{-n}$ and so $p(\nu, \mu) \leq 2^{-n}$. Thus $B_{d}(\mu, \epsilon) \subseteq B_{p}(\mu, \delta)$.

For the other direction, consider $B_{d}(\mu, \delta)$. Let $\epsilon=2^{-n}$ for some $n$ such that $2^{-n+1}<\delta$. Now if $\langle\sigma\rangle \leq n$ we have that $|\sigma| \leq n$ so that $[\sigma]^{\epsilon}=[\sigma]$. If $\nu \in B_{p}(\mu, \epsilon)$ we have that $|\mu(\sigma)-\nu(\sigma)|<\epsilon$ and so

$$
d(\mu, \nu) \leq \sum_{\langle\sigma\rangle \leq n} 2^{-\langle\sigma\rangle}|\mu(\sigma)-\nu(\sigma)|+2^{-n} \leq 2^{-n+1} .
$$

Thus $B_{p}(\mu, \epsilon) \subseteq B_{d}(\mu, \delta)$.

We will treat the space $\mathcal{P}\left(2^{\omega}\right)$ of probability measures as a computable metric space. These were introduced by Lacombe [7, though our presentation is influenced by [19]. A computable metric space is a triple $(\mathcal{X}, \mathcal{Q}, d)$, where $\mathcal{X}$ is a complete 
separable metric space, $\mathcal{Q}$ is an enumeration of a countable dense subset of $\mathcal{X}$, and $d$ is a metric computable on the elements of $\mathcal{Q}$. Given a computable metric space $(\mathcal{X}, \mathcal{Q}, d)$, with $\mathcal{Q}=\left\{q_{1}, q_{2}, \ldots\right\}$, the standard fast Cauchy representation of $(\mathcal{X}, \mathcal{Q}, d)$ is $\rho_{C}: 2^{\omega} \rightarrow \mathcal{X}$ and is defined by $\rho_{C}\left(0^{n(0)} 10^{n(1)} 10^{n(2)} 1 \ldots\right)=x$ if $(\forall i \in \omega)$ $d\left(x, q_{n(i)}\right) \leq 2^{-i}$. Note that this representation $\rho_{C}$ is a partial function.

In order to work with $\mathcal{P}\left(2^{\omega}\right)$ as a computable metric space, we need an enumeration of a countable dense subset of $\mathcal{P}\left(2^{\omega}\right)$ on which the metric is computable. For any $X \in 2^{\omega}$, we define the Dirac measure $\delta_{X}$ by

$$
\delta_{X}(\sigma)= \begin{cases}1 & \text { if } \sigma \prec X, \\ 0 & \text { otherwise }\end{cases}
$$

We will take our dense subset $\mathcal{Q}$ to be those measures that concentrate on sequences with finitely many $1 \mathrm{~s}$, and take rational values at those points. In other words, $\mu \in \mathcal{Q}$ if and only if $\mu=\sum_{i=1}^{n} a_{i} \delta_{\sigma_{i} 0^{\omega}}$, where $\sigma_{1}, \ldots, \sigma_{n} \in 2^{<\omega}$ and $a_{1}, \ldots, a_{n}$ are positive rationals such that $\sum_{i=1}^{n} a_{i}=1$.

Fix an enumeration of these measures $m_{1}, m_{2}, \ldots$ At times, in order to avoid subscripts, we will write $m(i)$ for $m_{i}$. Note that $d\left(m_{i}, m_{j}\right)$ is computable in $i$ and $j$ and that the open balls $B\left(m_{i}, 2^{-n}\right)$ form an enumerable basis for the topology on $\mathcal{P}\left(2^{\omega}\right)$. We will call these the $i d e a l$ open balls and take $B_{i}$ to be the $i$ th such ball in some fixed enumeration. Let $\overline{B_{i}}$ be the closure of the ideal ball $B_{i}$.

Instead of using the standard fast Cauchy representation of $\mathcal{P}\left(2^{\omega}\right)$, we want to use the fact that $\mathcal{P}\left(2^{\omega}\right)$ is compact to define a representation that has some additional useful properties. Reimann showed that there is a computable surjection $\rho: P \rightarrow \mathcal{P}\left(2^{\omega}\right)$, where $P$ is a $\Pi_{1}^{0}$ subset of $2^{\omega}[14$. Our approach is similar to that of Reimann. It can also be seen as a generalization of Turing's approach to coding the reals via overlapping intervals [18, for which he acknowledges Brouwer.

To define our representation, we will first define a Turing functional $\varphi$ such that $\varphi^{X}$ is total for all oracles $X$, and for all $n \in \omega$,

$$
d\left(m\left(\varphi^{X}(n)\right), m\left(\varphi^{X}(n+1)\right)\right) \leq 2^{-n} .
$$

Thus for any oracle $X$, the sequence $m\left(\varphi^{X}(0)\right), m\left(\varphi^{X}(1)\right), \ldots$ is Cauchy and so converges (because $\mathcal{P}\left(2^{\omega}\right)$ is complete). Thus we can define a total function $\rho: 2^{\omega} \rightarrow$ $\mathcal{P}\left(2^{\omega}\right)$ by $\rho(X)=\lim _{s} m\left(\varphi^{X}(s)\right)$.

We define $\varphi^{X}$ inductively as follows. At stage $s$ we will define $\varphi^{X}(s)$ for all oracles $X$. At stage 0 , we will define $\phi^{X}(0)=1$ for all oracles $X$. Note that $B\left(m_{1}, 2^{0}\right)=\mathcal{P}\left(2^{\omega}\right)$.

At stage $s+1$, for all strings $\tau$ such that $\varphi^{\tau}(s)$ is defined but $\varphi^{\tau^{\prime}}(s)$ is not defined, if $\tau^{\prime}$ is a strict initial segment of $\tau$ do the following. Let $m=m\left(\varphi^{\tau}(s)\right)$. We claim that we can uniformly compute a finite open covering $\left\{B\left(m\left(n_{1}\right), 2^{-s-1}\right), \ldots\right.$, $\left.B\left(m\left(n_{k}\right), 2^{-s-1}\right)\right\}$ of $\overline{B\left(m, 2^{-s}\right)}$ with $m\left(n_{i}\right) \in B\left(m, 2^{-s}\right)$. Given this claim, we can determine a disjoint collection of cylinders $\left\{\left[\tau_{1}\right], \ldots,\left[\tau_{k}\right]\right\}$ that covers $2^{\omega}$ and define $\varphi^{\tau \tau_{i}}(s+1)=n_{i}$. This completes the definition of $\varphi$. Our representation will be the continuous function $\rho: 2^{\omega} \rightarrow \mathcal{P}\left(2^{\omega}\right)$ defined by $\rho(X)=\lim _{s} m\left(\varphi^{X}(s)\right)$.

To establish the claim, we build a finite set of probability measures (all in $\mathcal{Q}$ ) by adding together measures of the form $2^{-s-4} \cdot \delta_{\sigma 0^{\omega}}$, where $|\sigma|=s+4$. Define

$$
S_{s}=\left\{m_{i} \in \mathcal{Q}: m_{i}=\sum_{|\sigma|=s+4} a_{\sigma} 2^{-s-4} \delta_{\sigma 0^{\omega}} \text { for some } a_{\sigma} \in \omega\right\} .
$$


Then we take $\left\{B\left(m_{i}, 2^{-s-1}\right): m_{i} \in S_{s} \wedge d\left(m, m_{i}\right)<2^{-s}\right\}$ as our covering. To show that this is in fact a covering, take any $\mu \in \overline{B\left(m, 2^{-s}\right)}$. Let $\nu=(m+3 \mu) / 4$, so $d(m, \nu)=3 d(m, \mu) / 4$ and $d(\mu, \nu)=d(m, \nu) / 4$. We can easily find $m_{i} \in S$ such that $d\left(\nu, m_{i}\right)<2^{-s-2}$. Hence the ball $B\left(m_{i}, 2^{-s-1}\right)$ is in our covering and this ball contains $\mu$.

Lemma 2.2. The function $\rho$ is total, surjective, and for all $X \in 2^{\omega}, \rho^{-1}(\rho(X))$ is a $\Pi_{1}^{0}(X)$ class.

Proof. We have already seen that $\rho$ is total. To see that it is surjective, take any $\mu \in \mathcal{P}\left(2^{\omega}\right)$. As $\rho$ is continuous, for all $n$, the set $F_{n}=\left\{X \in 2^{\omega}: d(\rho(X), \mu) \leq 2^{-n}\right\}$ is closed. The construction ensures that it is non-empty, so by compactness there is an $X \in \bigcap_{i} F_{i}$. Clearly, $\rho(X)=\mu$.

If $X \in 2^{\omega}$, then $\rho^{-1}(X)$ is a $\Pi_{1}^{0}(X)$ class because $Y \in \rho^{-1}(X)$ if and only if, for all $n$,

$$
d\left(m\left(\varphi^{X}(n)\right), m\left(\varphi^{Y}(n)\right)\right)<2^{-n+2} .
$$

Because $\rho$ is surjective, it is a representation of $\mathcal{P}\left(2^{\omega}\right)$. Furthermore, in the sense of representation theory, it is equivalent to the standard fast Cauchy representation $\rho_{C}$. Intuitively two representations are equivalent if we can computably convert between them. If $\rho(R)=\mu$, then $\rho(R)$ is just a fast Cauchy representation of $\mu$ (i.e., the sequence $\left.m\left(\varphi^{R}(0)\right), m\left(\varphi^{R}(1)\right), \ldots\right)$. On the other hand if $\rho_{C}(S)=\mu$, then we can determine a sequence $R$ such that $\rho(R)=\mu$ by running through the construction of $\varphi$. We start with $\tau_{0}=\lambda$. At each stage $s+1$ we compute a sufficiently close approximation to $\mu$ so that we can choose $\tau_{s+1} \succ \tau_{s}$ with $\mu \in$ $B\left(m\left(\varphi^{\tau_{s+1}}(s+1)\right), 2^{-s-1}\right)$.

We will need one additional nice property of $\rho$ : that the inverse image of the closure of an ideal open ball is also a $\Pi_{1}^{0}$ class.

Lemma 2.3. If $B(s, q)$ is an ideal ball in $\mathcal{P}\left(2^{\omega}\right)$, then $\rho^{-1}(\overline{B(s, q)})$ is a $\Pi_{1}^{0}$ subset of $2^{\omega}$.

Proof. First we show that $X \in \rho^{-1}(\overline{B(s, q)})$ if and only if, for all $i, j$,

$$
\rho(X) \in B\left(s_{i}, q_{j}\right) \text { implies } d\left(s_{i}, s\right) \leq q+q_{j} .
$$

If for some $i, j, \rho(X) \in B\left(s_{i}, q_{j}\right)$ and $d\left(s_{i}, s\right)>q+q_{j}$, then $q+q_{j}<d\left(s, s_{i}\right) \leq$ $d(s, \rho(X))+d\left(\rho(X), s_{i}\right)<d(s, \rho(X))+q_{j}$ and hence $X \notin \rho^{-1}(\overline{B(s, q)})$. Conversely, assume that $\rho(X) \in B\left(s_{i}, q_{j}\right)$ implies $d\left(s_{i}, s\right) \leq q+q_{j}$, for all $i, j$. Then $d(\rho(X), s) \leq$ $d\left(\rho(X), s_{i}\right)+d\left(s, s_{i}\right)<q+2 q_{j}$. As $\rho(X)$ is contained in arbitrarily small ideal balls, we have $d(\rho(X), s) \leq q$. Now the predicate $\rho(X) \in B\left(s_{i}, q_{j}\right)$ implies $d\left(s_{i}, s\right) \leq q+q_{j}$ is $\Pi_{1}^{0}$. This is true as $\rho(X) \in B\left(s_{i}, q_{j}\right)$ and $d\left(s_{i}, s\right)>q+q_{j}$ are both $\Sigma_{1}^{0}$.

We are now ready to prove Theorem 1.6. We start with the easier direction.

Lemma 2.4. If $X \in 2^{\omega}$ is $\mu$-random, then it is $\mu$-random for uniform tests.

Proof. Let $W$ be a c.e. set defining the under-graph of a universal uniform test $t$. Assume that $X$ is not $\mu$-random for uniform tests. Let $R$ be any representation of $\mu$. Build an $R$-test as follows. Let

$$
V_{n}=\left\{X \in 2^{\omega}: t(\mu, X)>2^{n}\right\} .
$$

Immediately we have that if $t(\mu, X)=\infty$, then $X \in \bigcap_{n \in \omega} V_{n}$. To show that $\left\{V_{n}\right\}_{n \in \omega}$ is an $R$-test, first observe that $2^{n} \mu\left(V_{n}\right) \leq \int t(\mu, X) d \mu \leq 1$, so $\mu\left(V_{n}\right) \leq$ 
$2^{-n}$. Secondly, we have $X \in V_{n}$ if and only if $t(\mu, X)>2^{n}$ if and only if, for some $\langle i, \sigma, q\rangle \in W$, we have $\mu \in B_{i}$ (which is c.e. in $R$ ), $X \in[\sigma]$ and $q>2^{n}$. Hence the $V_{n}$ are uniformly $\Sigma_{1}^{0}(R)$ sets, and $X$ is not $R$-random. As this holds for any representation of $\mu$, we have proved that $X$ is not $\mu$-random.

For the other direction, we have to show that the failure of $\mu$-randomness can be detected in a uniform way. Not surprisingly, we do this using the universal oracle Martin-Löf test $\left\{U_{n}\right\}_{n \in \omega}$ from above.

Lemma 2.5. If $X$ is not $\mu$-random, then for all $n$, there exists an $m$, such that for all $R \in \rho^{-1}\left(B\left(\mu, 2^{-m}\right)\right), X \in U_{n}^{R}$.

Proof. Take any $\mu \in \mathcal{P}\left(2^{\omega}\right)$. Assume that for some $n$, for all $m$, there is an $R_{m}$ such that $\rho\left(R_{m}\right) \in B\left(\mu, 2^{-m}\right)$ and $X \notin U_{n}^{R_{m}}$. Consider the tree $\left\{\sigma \in 2^{<\omega}:(\exists m) \sigma \preceq\right.$ $R_{m}\lceil m\}$. This tree is infinite, so it has an infinite path $A$. For all $i, \rho([A \uparrow i])$ includes the $\rho$ image of infinitely many $R_{m}$. The set $\rho([A \uparrow i])$ is closed because it is the continuous image of a compact set. Thus $\mu \in \rho([A\lceil i])$ and hence $\rho(A)=\mu$. But note that $X \notin U_{n}^{A}$, or otherwise $X \in U_{n}^{R_{m}}$ for some $m$. Thus $X$ must be $\mu$-random.

Proof of Theorem 1.6. Lemma 2.4 shows that if $X$ is not $\mu$-random for uniform tests, then $X$ is not $\mu$-random. To establish the other direction, we will construct a test $f$ as follows. For all $i$, let $K_{i}=\rho^{-1}\left(\overline{B_{i}}\right)$. By Lemma $2.3, K_{i}$ is a $\Pi_{1}^{0}$ class. So if $X$ enters $U_{n}^{R}$ for all $R$ in $K_{i}$, compactness ensures that we can determine this at some finite stage. If this occurs, then we can increase the value on some open set containing $X$ for all measures in $B_{i}$.

Given any c.e. set $W$, and any $s \in \omega$, we will let $W[s]$ be the set obtained by enumerating $W$ for $s$ steps. If $\tau$ is a finite string, we will let $U_{n}^{\tau}$ be the c.e. set obtained from $U_{n}^{X}$ (for any $X \succ \tau$ ) such that the elements enumerated into this set only make use of the oracle up to $|\tau|$.

Let $S_{1}, S_{2}, \ldots$ be an enumeration of all finite sets of finite strings. The undergraph of our test $f$ will be enumerated by the following c.e. set:

$$
W=\left\{\left\langle i, \sigma, 2^{n}\right\rangle:(\exists j)(\exists s) K_{i}[s] \subseteq \bigcup_{\tau \in S_{j}}[\tau] \text { and }[\sigma] \subseteq \bigcap_{\tau \in S_{j}} U_{2 n}^{\tau}[s]\right\} .
$$

Given any $\mu \in \mathcal{P}\left(2^{\omega}\right)$ we will show that $\int f(\mu, X) d \mu \leq 1$, so $f$ is a uniform test. Take $R \in \rho^{-1}(\mu)$. Take any $n$ and any $X \notin U_{2 n}^{R}$. Given any $i$, if $\mu \in B_{i}$, then $R \in K_{i}$. So if $S_{j}$ covers $K_{i}$, then for some $\tau \in S_{j}, \tau \prec R$. Thus $X \notin \bigcap_{\tau \in S_{j}} U_{2 n}^{\tau}$. This implies that $\left\langle i, \sigma, 2^{n}\right\rangle \notin W$ for any $\sigma \prec X$, and so $f(\mu, X) \leq 2^{n-1}$. Hence $f(\mu, X) \leq \max \left\{2^{n}: X \in U_{2 n}^{R}\right\}$ and so,

$$
\int f(\mu, X) d \mu \leq \sum_{i=1}^{\infty} 2^{i} \mu\left(U_{2 i}^{R}\right) \leq \sum_{i=1}^{\infty} 2^{i} 2^{-2 i}=1 .
$$

Now assume that $X$ is not $\mu$-random. Fix $n$. By Lemma 2.5, there is an $m$ such that if $R \in \rho^{-1}\left(B\left(\mu, 2^{-m}\right)\right)$, then $X \in U_{2 n}^{R}$. Let $\overline{B_{i}}$ be a closed ideal ball with $\mu \in \overline{B_{i}} \subseteq B\left(\mu, 2^{-m}\right)$. Now because for all $R \in K_{i}$ we have $X \in U_{2 n}^{R}$, the set $C=\left\{\tau \in 2^{<\omega}: X \in U_{2 n}^{\tau}\right\}$ is an open covering of $K_{i}$. Hence there is a finite subcovering $S$ of $C$ and a stage $s$ such that $S$ covers $K_{i}[s]$. So there is a $\sigma$ with $X \in[\sigma] \subseteq \bigcap_{\tau \in S} U_{2 n}^{\tau}[s]$. Thus $\left\langle i, \sigma, 2^{n}\right\rangle \in W$ and consequently $f(\mu, X)>2^{n}$. This holds for all $n$, so $f(\mu, X)=\infty$. Therefore, $X$ is not $\mu$-random for uniform tests. 
The anonymous referee has observed that Theorem 1.6 can be adapted to locally compact metric spaces, provided the compactness is suitably effective.

\section{Neutral measures}

Our main goal in the remainder of the paper will be to come to a better understanding of (weakly) neutral measures. The concept of a neutral measure was introduced by Levin [9, who proved their existence. The term neutral measure was introduced by Gács [3. As we will see in Section 4, where we derive several facts about neutral measures, it is often enough to assume a weaker property.

Definition 3.1. Let $\mu$ be a measure.

(i) $\mu$ is neutral for a uniform test $t$ if $(\forall X) t(\mu, X) \leq 1$.

(ii) $\mu$ is a neutral measure if it is neutral for some universal test.

(iii) $\mu$ is weakly neutral if every sequence is $\mu$-random.

Since a constant multiple of a universal test is also a universal test, and any two universal tests majorize each other up to a multiplicative constant, we can restate the second definition: $\mu$ is a neutral measure iff $(\exists c)(\forall X) t(\mu, X) \leq c$, where $t$ is any universal test.

It is immediate that a neutral measure $\mu$ is weakly neutral. Indeed, this is the property that makes neutral measures seem so unlikely. One might think that it is impossible for every sequence to be $\mu$-random, since if we have access to $\mu$, we should be able to build a Martin-Löf $\mu$-test covering something. Indeed, this is the case; in Lemma 4.1 we will see that for every representation $R$ of $\mu$, there is a non$R$-random sequence. But if $\mu$ is weakly neutral, no sequence will be de-randomized by every representation of $\mu$.

We start by giving a proof that neutral measures exist.

Theorem 3.2 (Levin [9]). For any uniform test, there is a measure neutral for it.

Our proof is fundamentally equivalent to that given by Levin [9] and Gács [3]. However, we will make use of the Kakutani fixed point theorem instead of Sperner's Lemma. Our exposition of the proof will also make clear some computability properties of neutral measures.

Theorem 3.3 (Kakutani [6]). If $\mathcal{S}$ is a non-empty compact convex subset of $\mathbb{R}^{n}$ and $\phi: \mathcal{S} \rightarrow \mathcal{S}$ a multi-valued map with closed graph and convex non-empty images, then there is an $x \in \mathcal{S}$ such that $x \in \phi(x)$.

A fixed point theorem is useful because a uniform test defines a map from measures to measures. Given $t$, we define $\hat{t}: \mathcal{P}\left(2^{\omega}\right) \rightarrow \mathcal{M}\left(2^{\omega}\right)$ by letting $\hat{t}(\mu)$ (which we will write as $\hat{t} \mu$ ) be the measure that takes the following values on the basic clopen sets:

$$
\hat{t} \mu(\sigma)=\frac{1}{2} \int_{[\sigma]} t(\mu, X) d \mu+\frac{1}{2} 2^{-|\sigma|} .
$$

We can partially order $\mathcal{M}\left(2^{\omega}\right)$ by $\mu \leq \nu$ if $\mu(\sigma) \leq \nu(\sigma)$ for all $\sigma$. In this case we will say that $\nu$ majorizes $\mu$.

Lemma 3.4. If $\mu \geq \hat{t} \mu$, then $\mu$ is neutral for $\frac{1}{2} \cdot t$. 
Proof. Assume $\mu$ is not neutral for $\frac{1}{2} \cdot t$. Then for some $X \in 2^{\omega}, t(\mu, X)>2$. This implies that there is a $\sigma \in 2^{<\omega}$ with $X \in[\sigma]$ such that $t(\mu, Y)>2$ for all $Y \in[\sigma]$. But this would mean that

$$
\hat{t} \mu(\sigma) \geq \frac{1}{2} \int_{[\sigma]} 2 d \mu+2^{-|\sigma|-1}>\mu(\sigma) .
$$

This is a contradiction because $\mu$ majorizes $\hat{t} \mu$.

Consider the sets $F_{n}=\left\{\mu \in \mathcal{P}\left(2^{\omega}\right):(\forall \sigma)|\sigma|=n \Rightarrow \hat{t} \mu(\sigma) \leq \mu(\sigma)\right\}$. Note that $\mathcal{P}\left(2^{\omega}\right)=F_{0}$ and $F_{n+1} \subseteq F_{n}$. Now if we can show that $F_{n}$ is non-empty and closed, for all $n$, then by the finite intersection property, there exists a $\mu \in \bigcap_{i \in \omega} F_{i}$. Thus $\mu$ has the property that $\mu \geq \hat{t} \mu$; hence it is neutral for $t$.

Lemma 3.5. For all $n$, the following is a $\Pi_{1}^{0}$ class:

$$
M_{n}=\left\{\left(R_{1}, R_{2}\right) \in 2^{\omega} \times 2^{\omega}:(\forall \sigma)|\sigma|=n \Rightarrow\left[\hat{t} \rho\left(R_{1}\right)\right](\sigma) \leq\left[\rho\left(R_{2}\right)\right](\sigma)\right\} .
$$

Proof. Take $R_{1}, R_{2} \in 2^{\omega}$ and let $\mu=\rho\left(R_{1}\right)$ and $\nu=\rho\left(R_{2}\right)$. Now $\left(R_{1}, R_{2}\right) \notin M_{n}$ if and only if, for some $\sigma$ of length $n$,

$$
\hat{t} \mu(\sigma)>\nu(\sigma) .
$$

Note that $\nu(\sigma)$ is computable in $R_{2}$ and $\hat{t} \mu(\sigma)$ is left c.e. in $R_{1}$. We can assume that $\nu(\sigma)$ is computed using an approximation from above (i.e., for all $s, \nu(\sigma)[s] \geq$ $\nu(\sigma)[s+1])$ and that $\nu(\sigma)[s]$ depends only on $R_{2}\lceil s$. We can also assume that $\hat{t} \mu(\sigma)[s]$ depends only on $R_{1} \uparrow s$. Then equation (3.1) holds if and only if for some $\sigma$ of length $n$ and stage $s$, we have $\hat{t} \mu(\sigma)[s]>\nu(\sigma)[s]$, and this allows us to expel $\left[R_{1} \oplus R_{2}\lceil 2 s]\right.$ from $M_{n}$.

Proposition 3.6. There is a measure $\mu \in \mathcal{P}\left(2^{\omega}\right)$ such that $\mu$ majorizes $\hat{t} \mu$.

Proof. As $M_{n}$ is closed, $F_{n}=\rho\left(\left\{R:(R, R) \in M_{n}\right\}\right)$ is closed. The only remaining task is to show that $F_{n}$ is non-empty. Let $S_{n}=\left\{\bar{x} \in[0,1]^{2^{n}}: \sum_{i=1}^{2^{n}} x_{i}=1\right\}$. Define a continuous map $\psi: S_{n} \rightarrow \mathcal{P}\left(2^{\omega}\right)$ by

$$
\psi(\bar{x})=\sum_{i=1}^{2^{n}} x_{i} \delta_{\sigma_{i} 0^{\omega}},
$$

where $\sigma_{i}$ is the $i$ th string of length $n$. Define the multi-valued map $\phi: S_{n} \rightarrow S_{n}$ by

$$
\phi(\bar{x})=\left\{\bar{y} \in S_{n}:\left(\forall i \leq 2^{n}\right) y_{i} \geq[\hat{t} \psi(\bar{x})]\left(\sigma_{i}\right)\right\} .
$$

Note that $y_{i}=[\psi(\bar{y})]\left(\sigma_{i}\right)$. So if $\bar{x}$ is a fixed point of $\phi$, then $\psi(\bar{x}) \in F_{n}$.

Define the function $\rho_{2}: 2^{\omega} \times 2^{\omega} \rightarrow \mathcal{P}\left(2^{\omega}\right) \times \mathcal{P}\left(2^{\omega}\right)$ by $\rho_{2}\left(R_{1}, R_{2}\right)=\left(\rho\left(R_{1}\right), \rho\left(R_{2}\right)\right)$, and the function $\psi_{2}: S_{n} \times S_{n} \rightarrow \mathcal{P}\left(2^{\omega}\right) \times \mathcal{P}\left(2^{\omega}\right)$ by $\psi_{2}(\bar{x}, \bar{y})=(\psi(\bar{x}), \psi(\bar{y}))$. Both $\rho_{2}$ and $\psi_{2}$ are continuous mappings. The graph of $\phi$ is precisely $\psi_{2}^{-1}\left(\rho_{2}\left(M_{n}\right)\right)$ and hence closed. Since $S_{n}$ is a non-empty compact convex subset of $\mathbb{R}^{2^{n}}$ and $\phi$ has non-empty convex images in $S_{n}$, Kakutani's theorem tells us that $\phi$ has a fixed point. Therefore, $F_{n}$ is non-empty.

This proposition establishes that the $\Pi_{1}^{0}$ class of Lemma 3.5 is not empty. Further if $t$ is a universal uniform test, then any representation in this $\Pi_{1}^{0}$ class is a representation of a neutral measure. In order to prove Theorem 3.2, we need one more application of compactness. 
Proof of Theorem 3.2. Given any $n \in \omega$, we could redefine $\hat{t} \mu$ by

$$
\hat{t} \mu(\sigma)=\frac{n}{n+1} \int_{[\sigma]} t(\mu, X) d \mu+\frac{1}{n+1} 2^{-|\sigma|} .
$$

The argument of Lemma 3.5 and Proposition 3.6 shows there exists a measure $\mu_{n}$ which is neutral for $\frac{n}{n+1} t$. This implies that if $n \neq 0$, then for all $X \in 2^{\omega}$ we have that $t\left(X, \mu_{n}\right) \leq \frac{n+1}{n}$. Because the space $\mathcal{P}\left(2^{\omega}\right)$ is compact, the sequence $\left\{\mu_{n}\right\}_{n \in \omega}$ has a convergent subsequence which converges to some measure $\mu$. If $\mu$ is not neutral for $t$, then there is some $X$ such that $t(\mu, X)>1$. This implies that for some open ball $B$ including $\mu$, and some $c>1$ we have that $t(\nu, X) \geq c$ for all $\nu \in B$. This is a contradiction because $B$ must include $\mu_{n}$ for some $n$ with $\frac{n+1}{n}<c$.

It is interesting that every known proof of the existence of a neutral measure uses a fixed point theorem or equivalent. Their existence seems to be a fundamentally topological fact. However, once we know such measures exist, they are relatively easy to find. There is a $\Pi_{1}^{0}$ class of (representations of) neutral measures, as we can take the intersection of the diagonals of the $\Pi_{1}^{0}$ classes $M_{n}$ when $t$ is a universal test. Recall that a Turing degree is a $P A$ degree if it can compute a member of every non-empty $\Pi_{1}^{0}$ subclass of $2^{\omega}$. So every PA degree computes a neutral measure. This lets us give a simple proof of the following theorem of Reimann and Slaman.

Theorem 3.7 (Reimann and Slaman [15]). For any $X \in 2^{\omega}, X$ is not computable if and only if there exists a representation $R$ of a measure such that $X$ is $R$-random and $\rho(R)$ does not concentrate on $X$.

Proof. If $X$ is computable, then consider any measure $\mu$ that does not concentrate on $X$. If $R$ is a representation of $\mu$, then it is simple to build an $R$-test that contains $X$ by finding initial segments of $X$ such that $\mu(X \uparrow n)$ is sufficiently small.

For the other direction, assume that $X$ is not computable. Then there is a $P$ of PA degree such that $P \Varangle_{T} X$. For example, Jockusch and Soare showed that there is a set of PA degree which is also of hyperimmune-free degree and another set of PA degree which is low, and hence these cannot both compute $X[5]$. As there is a $\Pi_{1}^{0}$ class of representations of neutral measures, $P$ computes a representation of some neutral measure $\mu$. Since $\mu$ is neutral, $X$ is $\mu$-random. Hence there exists a representation $R$ of $\mu$ (not necessarily computable from $P$ ) such that $X$ is $R$ random. Finally as $P$ cannot compute $X$, and $P$ can compute any atom of $\mu$, we know that $X$ is not an atom of $\mu$.

\section{Locating NeUtral measures}

In this section we study the computability-theoretic complexity of (weakly) neutral measures. In the previous section we noted that every PA degree computes a neutral measure. The reverse is true in a strong sense: if $\mu$ is a weakly neutral measure, then some PA degree is computable from every representation of $\mu$. As we will see, the story is complicated by the fact that weakly neutral measures themselves cannot have a Turing degree. We will show that their complexity can be measured using the continuous degrees, which were introduced by the second author. That will give us a better understanding of what is computable from (every representation of) a weakly neutral measure. We will prove that the ideal of Turing degrees below such a measure is a Scott ideal, and that every Scott ideal arises in 
this way. This, in turn, tells us about the atoms of weakly neutral measures (see Proposition 4.8).

One reason the existence of a weakly neutral measure may seem counterintuitive is that such a measure does not exist for representation tests.

Lemma 4.1. For all $R \in 2^{\omega}$, there exists an $X \in 2^{\omega}$ such that $X$ is not $R$-random.

Proof. Let $\mu=\rho(R)$. Construct an $R$-test as follows. Compute $\mu(\sigma)$ for all $\sigma$ of length 2 with precision $2^{-2}$. Take $\sigma_{1}$ to be the lexicographically least string of length 2 such that $\mu\left(\sigma_{1}\right)$ is within $\left[0,2^{-2}\right]$ for this level of precision. Let $V_{1}=\left[\sigma_{1}\right]$. Note that $\mu\left(\sigma_{1}\right) \leq 2^{-2}+2^{-2}=2^{-1}$. Once $V_{i}=\left[\sigma_{i}\right]$ has been defined with $\mu\left(\sigma_{i}\right) \leq 2^{-i}$, compute $\mu\left(\sigma_{i} \tau\right)$ for all $\tau$ of length 2 with precision $2^{-i-2}$. Take the lexicographically least $\tau$ such that $\mu\left(\sigma_{i} \tau\right) \leq 2^{-i-2}$ with this precision. Take $\sigma_{i+1}=\sigma_{i} \tau$, so $\mu\left(\sigma_{i+1}\right) \leq 2^{-i-1}$. Let $V_{i+1}=\left[\sigma_{i+1}\right]$. Thus $\bigcap_{i \in \omega} V_{i}$ is an $R$-test with non-empty intersection.

So for any representation $R$ of a weakly neutral measure $\mu$, there is an $X$ that is not $R$-random. However, there must be another representation $R^{\prime}$ of $\mu$ such that $X$ is $R^{\prime}$-random. The test constructed in the previous lemma cannot be made representation independent. The obstruction is that there is no canonical representation of a weakly neutral measure, and in fact, every representation contains extraneous information.

Theorem 4.2. A weakly neutral measure has no least Turing degree representation.

Proof. Let $\mu$ be a measure with least Turing degree representation $R$. By Lemma 4.1 there is an $R$-test that witnesses that some $X$ is not $R$-random. Let $R^{\prime}$ be any other representation of $\mu$. Since $R^{\prime}$ computes $R$, the $R$-test is also an $R^{\prime}$-test (as $R$ and $R^{\prime}$ represent the same measure). Hence $X$ is not $R^{\prime}$-random for any representation $R^{\prime}$ of $\mu$, and thus $X$ is not $\mu$-random. Therefore, $\mu$ is not weakly neutral.

If weakly neutral measures have no least Turing degree representation, then how should their computational power be examined? For this we turn to the continuous degrees introduced by Miller [12].

Definition 4.3. Let $\mathcal{M}_{0}$ and $\mathcal{M}_{1}$ be computable metric spaces and let $a \in \mathcal{M}_{0}$ and $b \in \mathcal{M}_{1}$. We define $a \leq_{r} b$ ( $a$ is representation reducible to $b$ ) if there is an index $e$ such that for every fast Cauchy representation $R$ of $b, \varphi_{e}^{R}$ is a fast Cauchy representation of $a$. The continuous degrees are the equivalence classes under $\equiv_{r}$.

Miller showed that the uniformity in the above definition is not required. In other words, it is equivalent to say that every fast Cauchy representation of $b$ computes a fast Cauchy representation of $a$, without fixing the index. This follows from the natural embedding of the continuous degrees into the enumeration degrees, see (4.1), and the fact that uniform and non-uniform enumeration reducibility are equivalent.

The finite sets are a countable dense subset of $2^{\omega}$ under the metric $d_{2^{\omega}}$ of Section 2. Thus for any $A \subseteq \omega$, we can talk about $\operatorname{deg}_{r}(A)$. This gives us an embedding of the Turing degrees into the continuous degrees. The continuous degrees that contain subsets of $\omega$ are the total degrees.

Using the fact that a continuous degree $a$ has total degree if and only if it has a least Turing degree representation [12, we obtain the following corollary to Theorem 4.2 
Corollary 4.4. Weakly neutral measures have non-total continuous degree.

This indicates that our study of (weakly) neutral measures can be enhanced by understanding the non-total continuous degrees. We start with the following definitions.

Definition 4.5. If $a$ and $b$ are Turing degrees, then $a$ is a $P A$ degree relative to $b(a \gg b)$ if every non-empty $\Pi_{1}^{0}(b)$ class contains a path computable from $a$. For $A, B \subseteq \omega$, we write $A \gg B$ to mean that $\operatorname{deg}_{T}(A) \gg \operatorname{deg}_{T}(B)$.

Definition 4.6. A non-empty countable class $S \subseteq 2^{\omega}$ is called a $S$ cott set if

(i) $A, B \in S$ implies that $A \oplus B \in S$,

(ii) $A \in S$ and $B \leq_{T} A$ implies $B \in S$, and

(iii) for every $A \in S$, there is a $B \in S$ such that $B \gg A$.

If $S$ is a Scott set, then $\left\{\operatorname{deg}_{T}(A): A \in S\right\}$ is a $S$ cott ideal.

We summarize some results of Miller. The Hilbert cube $[0,1]^{\omega}$ can be regarded as a computable metric space by using the metric defined at (2.1) along with the finitely non-zero sequences of rationals as a countable dense subset.

Theorem 4.7 (Miller [12]).

(i) Every continuous degree contains an element of $[0,1]^{\omega}$.

(ii) Let $a$ and $b$ be total degrees. Then $a \ll b$ if and only if there is a non-total degree $v$ with $a<_{r} v<_{r} b$.

(iii) The Turing ideal below a non-total continuous degree is a Scott ideal.

(iv) Any Scott ideal is the Turing ideal below some non-total continuous degree.

From Corollary 4.4 and Theorem 4.7(iii), we know that the ideal below any weakly neutral measure is a Scott ideal. One reason this is interesting is that understanding what can be computed from a weakly neutral measure is the same as understanding its atoms.

Proposition 4.8. $A \in 2^{\omega}$ is an atom of a weakly neutral measure $\mu$ if and only if $A \leq_{r} \mu$ (i.e., iff every representation of $\mu$ computes $A$ ).

Proof. Assume that every representation of $\mu$ computes $A$. If $\mu$ does not concentrate on $A$, then any representation $R$ of $\mu$ can compute an initial segment of $A$ with arbitrarily small $\mu$-measure, and hence capture $A$ in an $R$-test. Therefore, $A$ is not $\mu$-random and $\mu$ is not weakly neutral.

For the other direction, assume that $A$ is an atom of $\mu$ and let $R$ be any representation of $\mu$. Choose $\sigma \prec A$ such that $\mu(\sigma)<2 \mu(\{A\})$. Given $\sigma$, we can compute $A$ from $R$ by following the path consisting of all $\tau \succ \sigma$ such that $\mu(\sigma)<2 \mu(\tau)$. Therefore, $A \leq_{r} \mu$.

Every Scott ideal contains a PA degree, and hence contains a member of every non-empty $\Pi_{1}^{0}$ class. There is a $\Pi_{1}^{0}$ class containing only Martin-Löf random sequences; hence:

Corollary 4.9. Every weakly neutral measure has a Martin-Löf random atom.

This result allows us to answer a question of Gács [3, Question 1] in the negative. The question was speculative and, unfortunately, a negative answer does little more than shut down this speculation. The full context of the question would take too much space, but briefly, Gács was interested in capturing the mutual information of 
two sequences $X, Y \in 2^{\omega}$. Let $\mu$ be a neutral measure. Gács asked if it is would be reasonable to define the mutual information of $X$ and $Y$ as $\log t(\mu \times \mu, X \oplus Y)$. More specifically, he asked if this could, for the right choice of $\mu$, coincide with another definition he was considering. To see that this is not the case, let $A$ be a Martin-Löf random atom of $\mu$. Then $A \oplus A$ is an atom of $\mu \times \mu$; hence $\log t(\mu \times \mu, A \oplus A)$ must be finite. But a definition of mutual information that allows a Martin-Löf random sequence to have finite mutual information with itself is fairly absurd and, more concretely, behaves quite differently than other proposed definitions.

We have seen that the Turing ideal below a (weakly) neutral measure is always a Scott ideal. It turns out that the converse holds; the ideals below neutral measures are exactly the Scott ideals.

Theorem 4.10. Every Scott ideal is the ideal below some neutral measure.

To prove this theorem, we make further use of some prior work of Miller. To prove the existence of non-total continuous degrees, Miller developed the construction of a sequence $\alpha \in[0,1]^{\omega}$ that could not be diagonalized computably.

Definition 4.11. A sequence $\alpha \in[0,1]^{\omega}$ is diagonally non-computably diagonalizable, or d.n.c.d., if for all e, there exists a representation $R$ of $\alpha$ such that $\alpha(e)$ is an element of the convex closure of $\Pi_{e}(R)$ or $\Pi_{e}(R)$ is empty (where $\Pi_{e}(R)$ is the eth $\Pi_{1}^{0}(R)$ class $)$.

The convex closure of $\Pi_{e}(R)$ is $\left\{x \in[0,1]: \inf \Pi_{e}(R) \leq x \leq \sup \Pi_{e}(R)\right\}$. This definition of a d.n.c.d. sequence differs from that given in [12, but is equivalent up to continuous degree. The reason such sequences are referred to as diagonally non-computably diagonalizable is that if there is a Turing functional $\varphi$ and an $x$ such that $\varphi^{R}=x$ for any representation $R$ of $\alpha$, then (uniformly in the index of $\varphi)$ we can find an $e$ such that $\{x\}=\Pi_{e}(R)$ for all representations of $\alpha$. But then $\alpha(e)=x$. Thus $e$ witnesses the failure of $\varphi$ to diagonalize $\alpha$, uniformly in (the index for) $\varphi$. The last part of Theorem 4.7 can be strengthened to "any Scott ideal is the Turing ideal below some d.n.c.d. sequence".

To prove Theorem 4.10 we will show that any d.n.c.d. sequence is above, in the sense of $\geq_{r}$, a neutral measure that bounds the same total degrees. We will use semi-measures in our construction of this neutral measure. A semi-measure is a function $\tau: 2^{\omega} \rightarrow[0,1]$ such that $\tau(\sigma) \geq \tau(\sigma 0)+\tau(\sigma 1)$. We will identify a semi-measure $\tau$ with the set $S(\tau)=\left\{\langle\sigma, q\rangle \in 2^{<\omega} \times \mathbb{Q}: \tau(\sigma)>q\right\}$.

Semi-measures have been studied as computably enumerable objects; we call a semi-measure $\tau$ c.e. if $S(\tau)$ is c.e. Levin proved the existence of a universal c.e. semi-measure $\tau$, meaning that for every c.e. semi-measure $\tau^{\prime}$ there is a constant $c$ such that $\tau \geq c \tau^{\prime}[10$. This proof relativizes to show that for any set $A \subseteq \omega$, there is a universal c.e. in $A$ semi-measure. However, what does it mean to enumerate a semi-measure in some sequence $\alpha \in[0,1]^{\omega}$, if $\alpha$ does not have total degree? A reasonable suggestion would be to define a set to be c.e. in $\alpha$ if it is c.e. in every representation of $\alpha$. This can be easily expressed in terms of the enumeration degrees.

There is an embedding of the continuous degrees into the enumeration degrees. Given $\alpha \in[0,1]^{\omega}$ we define $\Xi(\alpha) \subseteq\{0,1\} \times \omega \times \mathbb{Q}$ by

$$
\Xi(\alpha)=\{\langle 0, i, q\rangle: q<\alpha(i)\} \cup\{\langle 1, i, q\rangle: q>\alpha(i)\} .
$$


If $R$ is a representation of $\alpha$, then $\Xi(\alpha)$ is c.e. in $R$. Further if $\Xi(\alpha)$ is c.e. in some set $B$, then $B$ Turing computes a representation of $\alpha$.

Now assume that a set $A$ is c.e. in $\alpha$ (i.e., c.e. in any representation of $\alpha$ ). Further, assume that $\Xi(\alpha)$ is c.e. in a set $B$. Since $B$ computes a representation of $\alpha$, it must be that $A$ is also c.e. in $B$. Hence $A \leq_{e} \Xi(\alpha)$. On the other hand, if $A \leq_{e} \Xi(\alpha)$, then as $\Xi(\alpha)$ is c.e. in any representation $R$ of $\alpha$, we have that $A$ is c.e. in any representation of $\alpha$. Thus the approach suggested above is equivalent to defining a semi-measure $\tau$ to be c.e. in $\alpha$ if $S(\tau) \leq_{e} \Xi(\alpha)$.

We can now provide a characterization of $\mu$-randomness in terms of the enumeration degrees. This is an extension of a result of Levin who proved the following theorem for the case that $\mu$ is a computable measure 8 .

Theorem 4.12. Take any $\mu \in \mathcal{P}\left(2^{\omega}\right)$. Then $X \in 2^{\omega}$ is $\mu$-random if and only if for every semi-measure $\tau$ c.e. in $\mu$ (i.e., $S(\tau) \leq_{e} \Xi(\mu)$ ), there exists $c \in \omega$ such that $\tau(\sigma) \leq c \mu(\sigma)$ for all $\sigma \prec X$.

Proof. If $X$ is not $\mu$-random, then for some uniform test, $t(\mu, X)=\infty$. Define a (semi-)measure $\tau=\hat{t} \mu$. Thus $\tau$ is c.e. in any representation of $\mu$. Fix $c$. Since $t(\mu, X)=\infty$, there is a $\sigma \prec X$ such that if $Y \in[\sigma]$, then $t(\mu, Y) \geq c$. Thus $\tau(\sigma)=\int_{[\sigma]} t(\mu, Y) d \mu \geq c \mu(\sigma)$.

For the other direction, assume that there is a semi-measure $\tau$ such that for all $c$, there exists a $\sigma \prec X$ with $\tau(\sigma)>c \mu(\sigma)$. Given any representation $R$ of $\mu$ we can enumerate $\tau$ and define a test $\left\{V_{i}\right\}_{i \in \omega}$ such that $V_{i}=\left\{[\sigma]: \tau(\sigma)>2^{i} \mu(\sigma)\right\}$. This test captures $X$, so $X$ is not $R$-random. As this is true for any representation of $\mu$, $X$ is not $\mu$-random.

This proof makes use of the equivalence between $\mu$-randomness and $\mu$-randomness for universal tests established in Theorem [1.6. Techniques developed by Hoyrup and Rojas can be used to construct a uniform test directly from a semimeasure providing an alternative proof of one direction [4].

We claim that, relative to a set $B$, we can enumerate all c.e. in $B$ semi-measures. Let $W_{e}(B)$ be the eth set c.e. in $B$ and let $W_{e, i}(B)$ be an enumeration of $W_{e}(B)$. Any set $X$ defines a weighting function $f_{X}(\sigma)=\sup \{q:\langle q, \sigma\rangle \in X\}$, where we are viewing $X$ as a subset of $\mathbb{Q} \times 2^{<\omega}$. Define $T_{e, 0}(B)=\emptyset$, and

$$
T_{e, i+1}(B)= \begin{cases}W_{e, i}(B) & \text { if } f_{W_{e, i}(B)} \text { is a semi-measure } \\ T_{e, i}(B) & \text { otherwise. }\end{cases}
$$

By passing from $T_{e}(B)$ to $S_{e}(B)=\left\{\left\langle q^{\prime}, \sigma\right\rangle:\langle q, \sigma\rangle \in T_{e}(B)\right.$ and $\left.q^{\prime} \leq q\right\}$, we get an effective list of exactly the c.e. in $B$ semi-measures.

To prove the following lemma, we use a representation $\rho: 2^{\omega} \rightarrow[0,1]^{\omega}$ with the same properties as the representation of $\mathcal{P}\left(2^{\omega}\right)$ constructed in Section 2. The same proof, mutatis mutandis, shows that such a representation exists.

Lemma 4.13. If $\alpha \in[0,1]^{\omega}$, then there is a universal semi-measure $\tau$ c.e. in $\alpha$.

Proof. Define $\hat{S}_{e}=\bigcap_{R \in \rho^{-1}(\alpha)} S_{e}(R)$. For any $R \in \rho^{-1}(\alpha)$, as $\rho^{-1}(\alpha)$ is $\Pi_{1}^{0}(R)$ class, it follows that $\hat{S}_{e}$ is c.e. in $R$. Let $\tau_{e}$ be $f_{\hat{S}_{e}}$. Note that this is a semi-measure. Define $\tau=\sum_{e=1}^{\infty} 2^{-e} \tau_{e}$. Let $S=S(\tau)$, so $S$ is c.e. in $R$ for all $R \in \rho^{-1}(\alpha)$.

Now if $\tau^{\prime}$ is a semi-measure c.e. in $\alpha$, then there is an index $e$ such that $\tau^{\prime}=S_{e}(R)$ for all $R \in \rho^{-1}(\alpha)$ (this holds because any reduction in the enumeration degrees 
is uniform, which is implicit in Selman [17 and proved independently by Rozinas [16]). Thus $\tau^{\prime}=\tau_{e}$, and so $\tau$ majorizes $\tau^{\prime}$.

Lemma 4.14. Let $\alpha \in[0,1]^{\omega}$ be a d.n.c.d. sequence. If $\tau$ is a semi-measure c.e. in $\alpha$, then there exists $\mu \in \mathcal{P}\left(2^{\omega}\right)$ such that $\mu \leq_{r} \alpha$ and $(\forall \sigma) \mu(\sigma) \geq \tau(\sigma)$.

Proof. We will define $\mu$ in such a way that any representation of $\alpha$ will (uniformly) be able to determine a representation of $\mu$. First define $\mu(\lambda)=1$. Hence $\mu(\lambda) \geq$ $\tau(\lambda)$.

Now assume that we have defined $\mu(\sigma)$ with $\mu(\sigma) \geq \tau(\sigma)$. Consider the interval $I_{\sigma}=[\tau(\sigma 0), \mu(\sigma)-\tau(\sigma 1)]$. Note that $I_{\sigma}$ is non-empty because $\mu(\sigma)-\tau(\sigma 1)-$ $\tau(\sigma 0) \geq \mu(\sigma)-\tau(\sigma) \geq 0$. Since $\tau(\sigma 0)$ and $\tau(\sigma 1)$ are left c.e. in $R$, and $\mu(\sigma)$ is computable in $R$, we see that $I_{\sigma}$ is a $\Pi_{1}^{0}(R)$ class. Further, everything is uniform, so we can actually compute an index $e$ such that $I_{\sigma}=\Pi_{e}(R)$, for any representation $R$ of $\alpha$. Because $\alpha$ is of d.n.c.d. degree, and $I_{\sigma}$ is its own convex closure, $\alpha(e) \in I_{\sigma}$. We define $\mu(\sigma 0)=\alpha(e)$ and $\mu(\sigma 1)=\mu(\sigma)-\mu(\sigma 0)$.

As $I_{\sigma}$ is non-empty, we have $\mu(\sigma 0) \leq \mu(\sigma)$, and $\mu(\sigma 0) \geq \tau(\sigma 0)$. Additionally, $\mu(\sigma 1)=\mu(\sigma)-\alpha(e) \geq \mu(\sigma)-\mu(\sigma)+\tau(\sigma 1)=\tau(\sigma 1)$.

We are finally ready to establish Theorem 4.10

Proof of Theorem 4.10, Let $\mathcal{I}$ be a Scott ideal. Let $\alpha$ be a d.n.c.d. sequence such that $\mathcal{I}$ is the Turing ideal below $\alpha$. Let $\tau$ be a universal semi-measure for $\alpha$. By Lemma 4.14, we can take $\mu \leq_{r} \alpha$ such that $\mu$ majorizes $\tau$ and $\mu \in \mathcal{P}\left(2^{\omega}\right)$. Let $t$ be a universal test. Since $\hat{t} \mu$ is a (semi-)measure c.e. in $\mu$, hence c.e. in $\alpha$, there is a $b$ such that $\hat{t} \mu \leq b \tau \leq b \mu$. So by Lemma 3.4, $\mu$ is neutral for the universal test $\frac{1}{b} t$.

If $A \in \mathcal{I}$, then $A \leq_{r} \alpha$. Any representation of $\alpha$ can compute $A$, so some semimeasure $\tau$ c.e. in $\alpha$ must concentrate on $A$. This means $A$ is an atom of $\mu$ and so $A \leq_{r} \mu$. If $A \leq_{r} \mu$, then $A \leq_{r} \alpha$, so $A \in \mathcal{I}$. Hence $\mathcal{I}$ is the Turing ideal below $\mu$.

The previous theorem appears to give a proof of the existence of neutral measures without using a fixed point theorem. However, this is not the case. Miller's construction of a d.n.c.d. sequence makes use of a generalization of the Kakutani fixed point theorem, and Lemma 4.14 makes essential use of this underlying fixed point theorem to construct the measure $\mu$.

\section{Open QUESTIONS}

Several questions remain open about the relationship between neutral measures and the continuous degrees. The most basic is:

Question 5.1. Does every non-total continuous degree contain a neutral measure?

In the proof of Theorem 4.10, we started with a d.n.c.d. sequence $\alpha$ and built a neutral measure $\mu \leq_{r} \alpha$ that bounds the same Turing degrees as $\alpha$. There is no reason to assume that $\mu \equiv_{r} \alpha$. While $\mu$ can list all of the elements of the sequence $\alpha$, it cannot necessarily determine the order of those elements. Even if we could improve the proof to show that $\mu \equiv_{r} \alpha$, we would run into another open question (from [12]):

Question 5.2. Does every non-total continuous degree contain a diagonally noncomputably diagonalizable sequence? 
If both questions are answered in the negative, it is natural to ask:

Question 5.3. Is there any relationship between the degrees of neutral measures and the degrees of d.n.c.d. sequences?

It is not too difficult to construct a weakly neutral measure that is not a neutral measure. For example, let $\mu$ be a neutral measure. Define $\nu$ such that:

(i) For all $\sigma \in 2^{<\omega}, \nu\left(0^{n} 1 \sigma\right)=2^{-n} \mu\left(0^{n} 1 \sigma\right)$.

(ii) $\nu$ has an atom at $0^{\omega}$.

The measure $\mu$ is weakly neutral because there is an atom at $0^{\omega}$ and every other sequence is in an open neighborhood where the measure looks neutral. However, there is a uniform test $t$ such that $t(\nu, X)=2^{n}$ if $X \in\left[0^{n} 1\right]$ (and of course, $\left.t\left(\nu, 0^{\omega}\right)=0\right)$. So $\nu$ is not a neutral measure.

The fact that these properties are different leads to natural questions:

Question 5.4. Is every weakly neutral measure representation equivalent to a neutral measure? Does every non-total continuous degree contain a weakly neutral measure?

\section{ACKNOWLEDGEMENTS}

The authors would like to thank Ian Haken for pointing out an error in the proof of Theorem 3.2 in an earlier version of this paper. The authors would also like to thank the anonymous referee of this paper for providing a number of helpful comments.

\section{REFERENCES}

[1] Patrick Billingsley. Convergence of Probability Measures. Wiley, 2nd edition, 1999. MR,1700749 (2000e:60008)

[2] R.G. Downey and D.R. Hirschfeldt. Algorithmic Randomness and Complexity. SpringerVerlag, New York, 2010. MR,2732288

[3] Peter Gács. Uniform test of algorithmic randomness over a general space. Theoret. Comput. Sci., 341(1-3):91-137, 2005. MR2159646(2006m:68057)

[4] Mathieu Hoyrup and Cristóbal Rojas. Computability of probability measures and Martin-Löf randomness over metric spaces. Inform. and Comput., 207(7):830-847, 2009. MR2519075 (2011b:03066)

[5] Carl G. Jockusch, Jr. and Robert I. Soare. $\Pi_{1}^{0}$ classes and degrees of theories. Trans. Amer. Math. Soc., 173:33-56, 1972. MR0316227 (47:4775)

[6] Shizuo Kakutani. A generalization of Brouwer's fixed point theorem. Duke Mathematical Journal, 8:457-459, 1941. MR0004776 (3:60c)

[7] Daniel Lacombe. Quelques procédés de définition en topologie recursive. In Constructivity in mathematics: Proceedings of the colloquium held at Amsterdam, 1957 (edited by A. Heyting), Studies in Logic and the Foundations of Mathematics, pages 129-158. NorthHolland Publishing Co., 1959. MR0112838(22:3687)

[8] L. A. Levin. The concept of a random sequence. Soviet Mathematics Doklady, 14(5):14131416, 1973. MR0366096 (51:2346)

[9] L. A. Levin. Uniform tests for randomness. Soviet Math. Dokl., 17(2):337-340, 1976. MR0414222 (54:2325)

[10] L.A. Levin and A.K. Zvonkin. The complexity of finite objects and the development of the concepts of information and randomness of means of the theory of algorithms. Russian Math. Surveys, 25(6), 1970. MR0307889(46:7004)

[11] P. Martin-Löf. The definition of random sequences. Information and Control, 9:602-619, 1966. MR0223179 (36:6228)

[12] Joseph S. Miller. Degrees of unsolvability of continuous functions. J. Symbolic Logic, 69(2):555-584, 2004. MR2058189(2005b:03102) 
[13] André Nies. Computability and randomness. Oxford University Press, 2009. MR 2548883 (2011i:03003)

[14] Jan Reimann. Effectively closed sets of measures and randomness. Ann. Pure Appl. Logic, 156(1):170-182, 2008. MR2474448 (2010a:03043)

[15] Jan Reimann and Theodore A. Slaman. Measures and their random reals. To appear.

[16] M. Rozinas. The semilattice of e-degrees. In Recursive functions (Russian), pages 71-84. Ivanov. Gos. Univ., Ivanovo, 1978. MR604944 (82i:03057)

[17] Alan L. Selman. Arithmetical reducibilities. I. Zeitschrift für Mathematische Logik und Grundlagen der Mathematik, 17:335-350, 1971. MR0304150 (46:3285)

[18] A. M. Turing. On computable numbers, with an application to the Entscheidungsproblem. A correction. Proceedings of the London Mathematical Society. Second Series, 43:544-546, 1937.

[19] Klaus Weihrauch. Computable analysis, an introduction. Springer-Verlag, Berlin, 2000. MR:1795407 (2002b:03129)

School of Mathematics, Statistics and Operations Research, Victoria University of Wellington, Wellington 6140, New Zealand

E-mail address: adam.day@msor.vuw.ac.nz

Department of Mathematics, University of Wisconsin, Madison, Wisconsin 53706-1388

E-mail address: jmiller@math.wisc.edu 